\title{
Solution of Singular and Nonsingular Initial and Boundary Value Problems by Modified Variational Iteration Method
}

\author{
Muhammad Aslam Noor and Syed Tauseef Mohyud-Din \\ Department of Mathematics, COMSATS Institute of Information Technology, Islamabad, Pakistan \\ Correspondence should be addressed to Muhammad Aslam Noor, noormaslam@hotmail.com
}

Received 19 May 2008; Accepted 5 June 2008

Recommended by Angelo Luongo

\begin{abstract}
We apply the modified variational iteration method (MVIM) for solving the singular and nonsingular initial and boundary value problems in this paper. The proposed modification is made by introducing Adomian's polynomials in the correct functional. The suggested algorithm is quite efficient and is practically well suited for use in such problems. The proposed iterative scheme finds the solution without any discretization, linearization, perturbation, or restrictive assumptions. Several examples are given to verify the efficiency and reliability of the suggested algorithm.
\end{abstract}

Copyright (C) 2008 M. A. Noor and S. T. Mohyud-Din. This is an open access article distributed under the Creative Commons Attribution License, which permits unrestricted use, distribution, and reproduction in any medium, provided the original work is properly cited.

\section{Introduction}

Many problems in applied sciences can be modeled by singular and nonsingular boundary value problems. The application of these problems involve astrophysics, experimental and mathematical physics, nuclear charge in heavy atoms, thermal behavior of a spherical cloud of gas, thermodynamics, population models, chemical kinetics, and fluid mechanics, see [1-46] and the references therein. Several techniques including decomposition, variational iteration, finite difference, polynomial spline, and homotopy perturbation have been developed for solving such problems, see [1-51] and the references therein. Most of these methods have their inbuilt deficiencies coupled with the major drawback of huge computational work. These facts have motivated to develop other methods for solving these problems. Adomian's decomposition method [42-44,51] was employed for finding solution of linear and nonlinear boundary value problems. He [12-18] developed the variational iteration method (VIM) for solving linear, nonlinear, initial, and boundary value problems. It is worth mentioning that the origin of variational iteration method can be traced back to Inokuti et al. [19]. In these 
methods, the solution is given in an infinite series usually converging to an accurate solution, see $[1-5,12-19,21,23,31-44,46,48,51]$ and the references therein. In this paper, we apply the modified variational iteration method (MVIM), which is obtained by the elegant coupling of variational iteration method and the Adomian's polynomials for solving singular and nonsingular initial and boundary value problems. This idea has been used by Abbasbandy $[1,2]$ implicitly, and by Noor and Mohyud-Din [36, 38, 40] for the solution of nonlinear boundary value problems. The basic motivation of this paper is to apply this modified variational iteration method (MVIM) for finding the solution of singular and nonsingular initial and boundary value problems. It is shown that the MVIM provides the solution in a rapid convergent series with easily computable components. We write the correct functional for the boundary value problem and calculate the Lagrange multiplier optimally. The Adomian's polynomials are introduced in the correct functional and evaluated by using the specific algorithm [42-44] and the references therein. Finally, the approximants are calculated by employing the Lagrange multipliers and the Adomian's polynomial scheme simultaneously. The use of Lagrange multiplier reduces the successive application of the integral operator and minimizes the computational work. Moreover, the selection of the initial value is done by exploiting the concept of modified decomposition method. In the present study, we apply this technique to solve boundary layer problem, unsteady flow of gas, singularly perturbed sixth-order Boussinesq, third-order dispersive, and fourth-order parabolic equations. To make the work more concise and to get a better understanding of the solution behavior, in case of boundary layer problem and the unsteady flow of gas, we replace the series solutions by the powerful Pade approximants [22, 28, 34, 43, 44, 47]. The use of Pade approximants shows real promise in solving boundary value problems in an infinite domain. The proposed MVIM solves effectively, easily, and accurately a large class of linear, nonlinear, partial, deterministic, or stochastic differential equations with approximate solutions which converge very rapidly to accurate solutions. Our results can be viewed as important and significant improvement of the previously known results.

\section{Variational iteration method}

To illustrate the basic concept of the technique, we consider the following general differential equation:

$$
L u+N u=g(x)
$$

where $L$ is a linear operator, $N$ a nonlinear operator, and $g(x)$ is the inhomogeneous term. According to variational iteration method $[1-5,13-19,21,23,31-41,46,48]$, we can construct a correct functional as follows:

$$
u_{n+1}(x)=u_{n}(x)+\int_{0}^{x} \lambda(s)\left(L u_{n}(s)+N \tilde{u}_{n}(s)-g(s)\right) d s,
$$

where $\lambda(s)$ is a Lagrange multiplier [13-18], which can be identified optimally via variational iteration method. The subscripts $n$ denote the $n$th approximation, $\tilde{u}_{n}$ is considered as a restricted variation, that is, $\delta \tilde{u}_{n}=0$. Relational (2.2) is called as a correct functional. The solution of the linear problems can be solved in a single iteration step due to the exact identification of the Lagrange multiplier. The principles of variational iteration method and its 
applicability for various kinds of differential equations are given in [13-18]. In this method, it is required first to determine the Lagrange multiplier $\lambda$ optimally. The successive approximation $u_{n+1}, n \geq 0$ of the solution $u$ will be readily obtained upon using the determined Lagrange multiplier and any selective function. Consequently, the solution is given by $u=\lim _{n \rightarrow \infty} u_{n}$. For the convergence criteria and error estimates of variational iteration method, see Ramos [41].

\section{Adomian's decomposition method}

To convey an idea of the technique, we consider the differential equation [42-44] of the form

$$
L u+R u+N u=g,
$$

where $L$ is the highest-order derivative which is assumed to be invertible, $R$ is a linear differential operator of order lesser than $L, N u$ represents the nonlinear terms, and $g$ is the source term. Applying the inverse operator $L^{-1}$ to both sides of (3.1) and using the given conditions, we obtain

$$
u=f-L^{-1}(R u)-L^{-1}(N u),
$$

where the function $f$ represents the terms arising from integrating the source term $g$ and by using the given conditions. Adomian's decomposition method [42-44] defines the solution by the series

$$
u(x)=\sum_{n=0}^{\infty} u_{n}(x)
$$

where the components $u_{n}(x)$ are usually determined recurrently by using the relation

$$
\begin{gathered}
u_{0}=f, \\
u_{k+1}=L^{-1}\left(R u_{k}\right)-L^{-1}\left(N u_{k}\right), \quad k \geq 0 .
\end{gathered}
$$

The nonlinear operator $N(u)$ can be decomposed into an infinite series of polynomials given by

$$
N(u)=\sum_{n=0}^{\infty} A_{n}
$$

where $A_{n}$ are the so-called Adomian's polynomials that can be generated for various classes of nonlinearities according to the specific algorithm developed in [42-44] which yields

$$
A_{n}=\left(\frac{1}{n !}\right)\left(\frac{d^{n}}{d \lambda^{n}}\right) N\left(\sum_{i=0}^{n}\left(\lambda^{i} u_{i}\right)\right)_{\lambda=0}, \quad n=0,1,2, \ldots
$$




\section{Modified variational iteration method (MVIM)}

To illustrate the basic concept of the variational decomposition method, we consider the following general differential (4.1), we have

$$
L u+N u=g(x)
$$

where $L$ is a linear operator, $N$ is a nonlinear operator, and $g(x)$ is the forcing term.

According to variational iteration method $[1-5,13-19,21,23,31-41,46,48]$, we can construct a correct functional as follows:

$$
u_{n+1}(x)=u_{n}(x)+\int_{0}^{x} \lambda\left(L u_{n}(s)+N \tilde{u}_{n}(s)-g(s)\right) d s,
$$

where $\lambda$ is a Lagrange multiplier [13-18], which can be identified optimally via variational iteration method. The subscripts $n$ denote the $n$th approximation, $\tilde{u}_{n}$ is considered as a restricted variation, that is, $\delta \tilde{u}_{n}=0(4.2)$ is called as a correct functional. We define the solution $u(x)$ by the series

$$
u(x)=\sum_{i=0}^{\infty} u_{i}(x)
$$

and the nonlinear term

$$
N(u)=\sum_{n=0}^{\infty} A_{n}\left(u_{0}, u_{1}, u_{2}, \ldots, u_{i}\right)
$$

where $A_{n}$ are the so-called Adomian's polynomials and can be generated for all types of nonlinearities according to the algorithm developed, in [42-44] which yields the following:

$$
A_{n}=\left(\frac{1}{n !}\right)\left(\frac{d^{n}}{d \lambda^{n}}\right) N(u(\lambda))
$$

Hence, we obtain the following iterative scheme for finding the approximate solution

$$
u_{n+1}(x)=u_{n}(x)+\int_{0}^{t} \lambda\left(L u_{n}(x)+\sum_{n=0}^{\infty} A_{n}-g(x)\right) d x
$$

which is called the modified variational iteration method (MVIM) and is formulated by the elegant coupling of variational iteration method and the Adomian's polynomials.

\section{Pade approximants}

A Pade approximant is the ratio of two polynomials constructed from the coefficients of the Taylor series expansion of a function $u(x)$. The $[L / M]$ Pade approximants to a function $y(x)$ are given by $[22,28,34,43,44,47]$

$$
\left[\frac{L}{M}\right]=\frac{P_{L}(x)}{Q_{M}(x)}
$$


where $P_{L}(x)$ is polynomial of degree at most $L$ and $Q_{M}(x)$ is a polynomial of degree at most $M$. The formal power series

$$
\begin{gathered}
y(x)=\sum_{i=1}^{\infty} a_{i} x^{i}, \\
y(x)-\frac{P_{L}(x)}{Q_{M}(x)}=O\left(x^{L+M+1}\right),
\end{gathered}
$$

determine the coefficients of $P_{L}(x)$ and $Q_{M}(x)$ by the equation. Since we can clearly multiply the numerator and denominator by a constant and leave $[L / M]$ unchanged, we imposed the normalization condition

$$
Q_{M}(0)=1.0 .
$$

Finally, we require that $P_{L}(x)$ and $Q_{M}(x)$ have non-common factors. If we write the coefficient of $P_{L}(x)$ and $Q_{M}(x)$ as

$$
\begin{aligned}
P_{L}(x) & =p_{0}+p_{1} x+p_{2} x^{2}+\cdots+p_{L} x^{L}, \\
Q_{M}(x) & =q_{0}+q_{1} x+q_{2} x^{2}+\cdots+q_{M} x^{M} .
\end{aligned}
$$

Then by (5.3) and (5.4), we may multiply (5.5) by $Q_{M}(x)$, which linearizes the coefficient equations. We can write out (5.5) in more details as

$$
\begin{gathered}
a_{L+1}+a_{L} q_{1}+\cdots+a_{L-M} q_{M}=0, \\
q_{L+2}+q_{L+1} q_{1}+\cdots+a_{L-M+2} q_{M}=0, \\
\vdots \\
a_{L+M}+a_{L+M-1} q_{1}+\cdots+a_{L} q_{M}=0, \\
a_{0}=p_{0} \\
a_{0}+a_{0} q_{1}+\cdots+=p_{1} \\
\vdots \\
a_{L}+a_{L-1} q_{1}+\cdots+a_{0} q_{L}=p_{L} .
\end{gathered}
$$

To solve these equations, we start with (5.5), which is a set of linear equations for all the unknown $q$ 's. Once the $q^{\prime}$ s are known, then (5.6) gives an explicit formula for the unknown $p^{\prime}$ s, which complete the solution. If (5.5) and (5.6) are nonsingular, then we can solve them directly and obtain (5.7) [22], where (5.7) holds, and if the lower index on a sum exceeds the upper, the sum is replaced by zero:

$$
\left[\frac{L}{M}\right]=\frac{\operatorname{det}\left[\begin{array}{cccc}
a_{L-M+1} & a_{L-M+2} & \cdots & a_{L+1} \\
\vdots & \vdots & \ddots & \vdots \\
a_{L} & a_{L+1} & \cdots & a_{L+M} \\
\sum_{j=M}^{L} a_{j-M} x^{j} & \sum_{j=M-1}^{L} a_{j-M+1} x^{j} & \cdots & \sum_{j=0}^{L} a_{j} x^{j}
\end{array}\right]}{\operatorname{det}\left[\begin{array}{cccc}
a_{L-M+1} & a_{L-M+2} & \cdots & a_{L+1} \\
\vdots & \vdots & \ddots & \\
a_{L} & a_{L+1} & \cdots & a_{L+M} \\
x^{M} & x^{M-1} & \cdots & 1
\end{array}\right]} .
$$

To obtain diagonal Pade approximants of different order such as [2/2], [4/4], or [6/6], we can use the symbolic calculus software Maple. 


\section{Numerical applications}

In this section, we apply the modified variational iteration method (MVIM) for solving the singular and nonsingular boundary value problems. We write the correct functional for the boundary value problem and carefully select the initial value because the approximants are heavily dependant on the initial value. The Adomian's polynomials are introduced in the correct functional for the nonlinear terms. The results are very encouraging indicating the reliability and efficiency of the proposed method. We apply the MVIM for solving the boundary layer problem; unsteady flow of gas through a porous medium; Boussinesq equations, third-order dispersive, and fourth-order parabolic singular partial differential equations. The powerful Pade approximants are applied in case of boundary-layer problem and unsteady flow in order to make the work more concise and for better understanding of the solution behavior.

Example 6.1 (see [43]). Consider the following nonlinear third-order boundary layer problem which appears mostly in the mathematical modeling of physical phenomena in fluid mechanics $[43,45]$ :

$$
f^{\prime \prime \prime}(x)+(k-1) f(x) f^{\prime \prime}(x)-2 n\left(f^{\prime}(x)\right)^{2}=0, \quad k>0,
$$

with boundary conditions

$$
f(0)=0, \quad f^{\prime}(0)=1, \quad f^{\prime}(\infty)=0, \quad k>0 .
$$

The correct functional is given as

$$
f_{n+1}(x)=f_{n}(x)+\int_{0}^{x} \lambda(s)\left(f_{n}^{\prime \prime \prime}(s)+(k-1) \tilde{f}_{n}(x) \tilde{f}_{n}^{\prime \prime}(s)-2 n\left(\tilde{f}_{n}^{\prime}(s)\right)^{2}\right) d s=0, \quad k>0 .
$$

Making the correct functional stationary, the Lagrange multipliers can be identified as $\lambda(s)=$ $-(1 / 2 !)(s-x)^{2}$, consequently, we have

$$
f_{n+1}(x)=f_{n}(x)-\int_{0}^{x} \frac{1}{2 !}(s-x)^{2}\left(f_{n}^{\prime \prime \prime}(s)+(k-1) \tilde{f}_{n}(s) \tilde{f}_{n}^{\prime \prime}(x)-2 n\left(\tilde{f}_{n}^{\prime}(s)\right)^{2}\right) d s=0, \quad k>0,
$$

where $f^{\prime \prime}(0)=\alpha<0$. Applying the modified variational iteration method, we have

$$
f_{n+1}(x)=f_{n}(x)+\int_{0}^{x} \frac{1}{2 !}(s-x)^{2}\left(f_{n}^{\prime \prime \prime}(s)+(k-1) \sum_{n=0}^{\infty} A_{n}-2 n \sum_{n=0}^{\infty} B_{n}\right) d s=0,
$$


Table 1: Numerical values for $\alpha=f^{\prime \prime}(0)$ for $0<k<1$ by using diagonal Pade approximants [43].

\begin{tabular}{lccccc}
\hline$n$ & {$[2 / 2]$} & {$[3 / 3]$} & {$[4 / 4]$} & {$[5 / 5]$} & {$[6 / 6]$} \\
\hline 0.2 & -0.3872983347 & -0.3821533832 & -0.3819153845 & -0.3819148088 & -0.3819121854 \\
$1 / 3$ & -0.5773502692 & -0.5615999244 & -0.5614066588 & -0.5614481405 & -0.561441934 \\
0.4 & -0.6451506398 & -0.6397000575 & -0.6389732578 & -0.6389892681 & -0.6389734794 \\
0.6 & -0.8407967591 & -0.8393603021 & -0.8396060478 & -0.8395875381 & -0.8396056769 \\
0.8 & -1.007983207 & -1.007796981 & -1.007646828 & -1.007646828 & -1.007792100 \\
\hline
\end{tabular}

where $A_{n}$ and $B_{n}$ are the so-called Adomian's polynomials and can be generated for all types of nonlinearities according to the algorithm defined in [42-44]. Consequently the following approximants are made:

$$
\begin{aligned}
f_{0}(x)= & x \\
f_{1}(x)= & x+\frac{1}{2} \alpha x^{2}+\frac{1}{3} x^{3} \\
f_{2}(x)= & x+\frac{1}{2} \alpha x^{2}+\frac{1}{3} x^{3}+\frac{1}{24} \alpha(3 n+1) x^{4}+\frac{1}{30} n(n+1) x^{5} \\
f_{3}(x)= & x+\frac{1}{2} \alpha x^{2}+\frac{1}{3} x^{3}+\frac{1}{24} \alpha(3 n+1) x^{4}+\frac{1}{30} n(n+1) x^{5}+\frac{1}{120} \alpha^{2}(3 n+1) x^{5} \\
& +\frac{1}{720} \alpha\left(19 n^{2}+18 n+3\right) x^{6}+\frac{1}{315} n\left(2 n^{2}+2 n+1\right) x^{7}, \\
f_{4}(x)= & x+\frac{1}{2} \alpha x^{2}+\frac{1}{3} x^{3}+\frac{1}{24} \alpha(3 n+1) x^{4}+\frac{1}{30} n(n+1) x^{5}+\frac{1}{120} \alpha^{2}(3 n+1) x^{5} \\
& +\frac{1}{720} \alpha\left(19 n^{2}+18 n+3\right) x^{6}+\frac{1}{315} n\left(2 n^{2}+2 n+1\right) x^{7}, \frac{1}{5040} \alpha^{2}\left(27 n^{2}+42 n+11\right) x^{7} \\
& +\frac{1}{40320} \alpha\left(167 n^{3}+297 n^{2}+161 n+15\right) x^{8}+\frac{1}{22680} n\left(13 n^{3}+38 n^{2}+23 n+6\right) x^{9},
\end{aligned}
$$

The series solution is given as

$$
\begin{aligned}
f(x)= & x+\frac{\alpha x^{2}}{2}+\frac{n x^{3}}{3}+\left(\frac{1}{8} n \alpha+\frac{1}{24} \alpha\right) x^{4}+\left(\frac{1}{30} n^{2}+\frac{1}{40} n \alpha^{2}+\frac{1}{120} \alpha^{2}+\frac{1}{30} n\right) x^{5} \\
& +\left(\frac{19}{720} n^{2} \alpha+\frac{1}{240} \alpha+\frac{1}{40} n \alpha\right) x^{6}+\left(\frac{1}{120} n \alpha^{2}+\frac{1}{315} n+\frac{2}{315} n^{3}+\frac{11}{5040} \alpha^{2}+\frac{3}{560} n^{2} \alpha^{2}+\frac{2}{315} n^{2}\right) x^{7} \\
& +\left(\frac{11}{40320} \alpha^{3}+\frac{33}{4480} n^{2} \alpha+\frac{3}{4480} \alpha^{3} n^{2}+\frac{23}{5760} n \alpha+\frac{1}{2688} \alpha+\frac{167}{40320} n^{3} \alpha+\frac{1}{960} \alpha^{3} n\right) x^{8} \\
& +\left(\frac{1}{3780} n+\frac{527}{362880} n^{3} \alpha^{2}+\frac{19}{11340} n^{3}+\frac{709}{362880} n \alpha^{2}+\frac{23}{8064} n^{2} \alpha^{2}+\frac{23}{22680} n^{2}+\frac{13}{22680} n^{4}\right. \\
& \left.+\frac{43}{120960} \alpha^{2}\right) x^{9}+\cdots .
\end{aligned}
$$

Example 6.2 (see [34, 44]). Consider the following nonlinear differential equation which governs the unsteady flow of gas through a porous medium:

$$
y^{\prime \prime}(x)+\frac{2 x}{\sqrt{1-\alpha y}} y^{\prime}(x)=0, \quad 0<\alpha<1 .
$$


Table 2: Numerical values for $\alpha=f^{\prime \prime}(0)$ for $k>1$ by using diagonal Pade approximants [43].

\begin{tabular}{lc}
\hline$n$ & $\alpha$ \\
\hline 4 & -2.483954032 \\
10 & -4.026385103 \\
100 & -12.84334315 \\
1000 & -40.65538218 \\
5000 & -104.8420672 \\
\hline
\end{tabular}

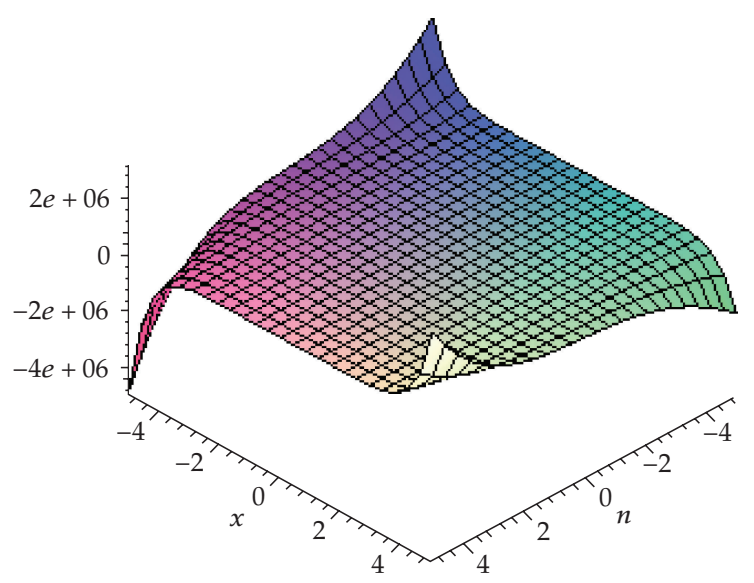

Figure 1: $(\alpha=-2.483954032)$.

With the following typical boundary conditions imposed by the physical properties [34, 44],

$$
y(0)=1, \quad \lim _{x \rightarrow \infty} y(x)=0 .
$$

The correct functional is given as

$$
y_{n+1}(x)=y_{n}(x)+\int_{0}^{x} \lambda(s)\left(y^{\prime \prime}(s)+\frac{2 x}{\sqrt{1-\alpha y}} y^{\prime}(s)\right) d s, \quad 0<\alpha<1 .
$$

Making the correct functional stationary, using $\lambda=x-s$, as the Lagrange multiplier, we get the following iterative formula:

$$
y_{n+1}(x)=y_{n}(x)+\int_{0}^{x}(s-x)\left(y^{\prime \prime}(s)+\frac{2 x}{\sqrt{1-\alpha y}} y^{\prime}(s)\right) d s, \quad 0<\alpha<1,
$$

where

$$
A=y^{\prime}(0)
$$

Applying the modified variational iteration method, we have

$$
y_{n+1}(x)=y_{n}(x)+\int_{0}^{x}(s-x)\left(y^{\prime \prime}(s)+2 x \sum_{n=0}^{\infty} A_{n}\right) d s, \quad 0<\alpha<1,
$$


where $A_{n}$ are the so-called Adomian's polynomials and can be generated for all types of nonlinearities according to the algorithm defined in [42-44]. First few Adomian's polynomials are as under:

$$
\begin{aligned}
A_{0}= & \left(1-\alpha y_{0}\right)^{-1 / 2} y_{0}^{\prime}, \\
A_{1}= & \left(1-\alpha y_{0}\right)^{-1 / 2} y_{1}^{\prime}+\frac{\alpha}{2}\left(1-\alpha y_{0}\right)^{-3 / 2} y_{0}^{\prime} y_{1}, \\
A_{2}= & \left(1-\alpha y_{0}\right)^{-1 / 2} y_{2}^{\prime}+\frac{\alpha}{2}\left(1-\alpha y_{0}\right)^{-3 / 2} y_{1}^{\prime} y_{1}+\frac{\alpha}{2}\left(1-\alpha y_{0}\right)^{-3 / 2} y_{0}^{\prime} y_{2}+\frac{3}{8} \alpha^{2}\left(1-\alpha y_{0}\right)^{-5 / 2} y_{0}^{\prime} y_{1}^{2}, \\
A_{3}= & \left(1-\alpha y_{0}\right)^{-1 / 2} y_{3}^{\prime}+\frac{\alpha}{2}\left(1-\alpha y_{0}\right)^{-3 / 2} y_{2}^{\prime} y_{1}+\frac{\alpha}{2}\left(1-\alpha y_{0}\right)^{-3 / 2} y_{1}^{\prime} y_{2}+\frac{\alpha}{2}\left(1-\alpha y_{0}\right)^{-3 / 2} y_{0}^{\prime} y_{3} \\
& +\frac{3}{8} \alpha^{2}\left(1-\alpha y_{0}\right)^{-5 / 2} y_{1}^{\prime} y_{1}^{2}+\frac{3}{4} \alpha^{2}\left(1-\alpha y_{0}\right)^{-5 / 2} y_{0}^{\prime} y_{1} y_{2}+\frac{5}{16} \alpha^{3}\left(1-\alpha y_{0}\right)^{-7 / 2} y_{0}^{\prime} y_{1}^{3},
\end{aligned}
$$

Consequently, the following approximants are obtained:

$$
\begin{aligned}
& y_{0}(x)= 1 \\
& y_{1}(x)= 1+A x, \\
& y_{2}(x)= 1+A x-\frac{A}{3 \sqrt{1-\alpha}} x^{3}, \\
& y_{3}(x)=1+A x-\frac{A}{3 \sqrt{1-\alpha}} x^{3}-\frac{\alpha A^{2}}{12(1-\alpha)^{3 / 2}} x^{4}+\frac{A}{10(1-\alpha)} x^{5}, \\
& y_{4}(x)=1+A x-\frac{A}{3 \sqrt{1-\alpha}} x^{3}-\frac{\alpha A^{2}}{12(1-\alpha)^{3 / 2}} x^{4}+\frac{A}{10(1-\alpha)} x^{5}-\frac{3 \alpha^{2} A^{3}}{80(1-\alpha)^{5 / 2}} x^{5} \\
&+\frac{\alpha A^{2}}{15(1-\alpha)^{2}} x^{6}+O\left(x^{7}\right), \\
& y_{5}(x)=1+A x-\frac{A}{3 \sqrt{1-\alpha}} x^{3}-\frac{\alpha A^{2}}{12(1-\alpha)^{3 / 2}} x^{4}+\frac{A}{10(1-\alpha)} x^{5}-\frac{3 \alpha^{2} A^{3}}{80(1-\alpha)^{5 / 2}} x^{5}+\frac{\alpha A^{2}}{15(1-\alpha)^{2}} x^{6} \\
&-\frac{\alpha^{3} A^{4}}{48(1-\alpha)^{7 / 2}} x^{6}+O\left(x^{7}\right),
\end{aligned}
$$

The series solution is given as

$$
\begin{aligned}
y(x)= & 1+A x-\frac{A}{3 \sqrt{1-\alpha}} x^{3}-\frac{\alpha A^{2}}{12(1-\alpha)^{3 / 2}} x^{4}+\left(\frac{A}{10(1-\alpha)}-\frac{3 \alpha^{2} A^{3}}{80(1-\alpha)^{5 / 2}}\right) x^{5} \\
& +\left(\frac{\alpha A^{2}}{15(1-\alpha)^{2}}-\frac{\alpha^{3} A^{4}}{48(1-\alpha)^{7 / 2}}\right) x^{6}+O\left(x^{7}\right) .
\end{aligned}
$$

Now, we investigate the mathematical behavior of the solution $y(x)$ in order to determine the 
Table 3: The initial slopes $A=y^{\prime}(0)$ for various values of $\alpha$.

\begin{tabular}{lcc}
\hline$\alpha$ & $B_{[2 / 2]}=y^{\prime}(0)$ & $B_{[3 / 3]}=y^{\prime}(0)$ \\
\hline 0.1 & -3.556558821 & -1.957208953 \\
0.2 & -2.441894334 & -1.786475516 \\
0.3 & -1.928338405 & -1.478270843 \\
0.4 & -1.606856838 & -1.231801809 \\
0.5 & -1.373178096 & -1.025529704 \\
0.6 & -1.185519607 & -0.8400346085 \\
0.7 & -1.021411309 & -0.6612047893 \\
0.8 & -0.8633400217 & -0.4776697286 \\
0.9 & -0.6844600642 & -0.2772628386 \\
\hline
\end{tabular}

initial slope $y^{\prime}(0)$. This goal can be achieved by forming diagonal Pade approximants [34, 44, 47] which have the advantage of manipulating the polynomial approximation into a rational function to gain more information about $y(x)$. It is well-known that Pade approximants will converge on the entire real axis $[20,22,28,34,43,44,47]$, if $y(x)$ is free of singularities on the real axis. It is of interest to note that Pade approximants give results with no greater error bounds than approximation by polynomials. More importantly, the diagonal approximant is the most accurate approximant; therefore we will construct only the diagonal approximants in the following discussions. Using the boundary condition $y(\infty)=0$, the diagonals approximant $[M / M]$ vanishes if the coefficient of $x$ with the highest power in the numerator vanishes. The computational work can be performed by using the mathematical software MAPLE. The physical behavior indicates that $y(x)$ is a decreasing function, hence $y^{\prime}(0)<0$. Using this fact, and following $[20,34,44]$, complex roots and nonphysical positive roots should be excluded. Based on this, the [2/2] Pade approximant produced the slope $A$ to be

$$
A=-\frac{2(1-\alpha)^{1 / 4}}{\sqrt{3 \alpha}}
$$

and using [3/3] Pade approximants we find

$$
A=-\frac{\sqrt{(-4674 \alpha+8664) \sqrt{1-\alpha}-144 \gamma}}{57 \alpha}
$$

where

$$
\gamma=\sqrt{5(1-\alpha)\left(1309 \alpha^{2}-2280 \alpha+1216\right)} .
$$

Using (6.17)-(6.19) gives the values of the initial slope $A=y^{\prime}(0)$ listed in Table 1 . The formulas (6.17) and (6.19) suggest that the initial slope $A=y^{\prime}(0)$ depends mainly on the parameter $\alpha$, where $0<\alpha<1$. Table 3 shows that the initial slope $A=y^{\prime}(0)$ increases with the increase of $\alpha$. The mathematical structure of $y(x)$ was successfully enhanced by using the Pade approximants. Table 4 indicates the values of $y(x)[34,44]$ and by using the [2/2] and [3/3] approximants for specific value of $\alpha=0.5$. 
Table 4: The values of $y(x)$ for $\alpha=0.5$ for $x=0.1$ to 1.0 .

\begin{tabular}{lccc}
\hline$X$ & $y$ kidder & $y_{[2 / 2]}$ & $y_{[3 / 3]}$ \\
\hline 0.1 & 0.8816588283 & 0.8633060641 & 0.8979167028 \\
0.2 & 0.7663076781 & 0.7301262261 & 0.7985228199 \\
0.3 & 0.6565379995 & 0.6033054140 & 0.7041129703 \\
0.4 & 0.5544024032 & 0.4848898717 & 0.6165037901 \\
0.5 & 0.4613650295 & 0.3761603869 & 0.5370533796 \\
0.6 & 0.3783109315 & 0.2777311628 & 0.4665625669 \\
0.7 & 0.3055976546 & 0.1896843371 & 0.4062426033 \\
0.8 & 0.2431325473 & 0.1117105165 & 0.3560801699 \\
0.9 & 0.1904623681 & 0.04323673236 & 0.3179966614 \\
1.0 & 0.1587689826 & 0.01646750847 & 0.2900255005 \\
\hline
\end{tabular}

Example 6.3 (see $[32,51])$. Consider the following singularly perturbed sixth-order Boussinesq equation:

$$
u_{t t}=u_{x x}+(p(u))_{x x}+\alpha u_{x x x x}+\beta u_{x x x x x x},
$$

taking $\alpha=1, \beta=0$, and $p(u)=3 u^{2}$, the model equation is given as

$$
u_{t t}=u_{x x}+3\left(u^{2}\right)_{x x}+u_{x x x x}
$$

with initial conditions

$$
u(x, 0)=\frac{2 a k^{2} e^{k x}}{\left(1+a e^{k x}\right)^{2}}, \quad u_{t}(x, 0)=\frac{2 a k^{3} \sqrt{1+k^{2}}\left(1-a e^{k x}\right) e^{k x}}{\left(1+a e^{k x}\right)^{3}},
$$

where $a$ and $k$ are arbitrary constants. The exact solution $u(x, t)$ of the problem is given as $[32,51]$

$$
u(x, t)=2 \frac{a k^{2} \exp \left(k x+k \sqrt{1+k^{2} t}\right)}{\left(1+a \exp \left(k x+k \sqrt{1+k^{2} t}\right)\right)^{2}} .
$$

The correct functional is given as

$$
\begin{aligned}
u_{n+1}(x, t)= & \frac{2 a k^{2} e^{k x}}{\left(1+a e^{k x}\right)^{2}}+\frac{2 a k^{3} \sqrt{1+k^{2}}\left(1-a e^{k x}\right) e^{k x}}{\left(1+a e^{k x}\right)^{3}} t \\
& +\int_{0}^{t} \lambda\left(\frac{\partial^{2} u_{n}}{\partial t^{2}}-\left(\tilde{u}_{n}\right)_{x x}-\left(\tilde{u}_{n}\right)_{x x x x}-3 \sum_{n=0}^{\infty} B_{n}\right) d t,
\end{aligned}
$$

where $B_{n}$ are Adomian's polynomials for nonlinear operator $F(u)=u^{2}(x)$ and can be generated for all types of nonlinearities according to the algorithm developed in $[42-44,51]$ which yields the following:

$$
\begin{aligned}
& B_{0}=\left(u_{0}^{2}\right)_{x x^{\prime}} \\
& B_{1}=2 u_{0} u_{1 x x}+4 u_{0 x} u_{1 x}+2 u_{0 x x} u_{1,} \\
& B_{2}=2 u_{0} u_{2 x x}+4 u_{0 x} u_{2 x}+2 u_{0 x x} u_{2}+2 u_{1 x} u_{1 x x}+2\left(u_{1 x}\right)^{2} \\
& B_{3}=\left(2 u_{0} u_{3}+2 u_{1} u_{2}\right)_{x x^{\prime}}
\end{aligned}
$$


Making the correct functional stationary, the Lagrange multiplier can be identified as $\lambda=x-t$, consequently,

$$
\begin{aligned}
u_{n+1}(x, t)= & \frac{2 a k^{2} e^{k x}}{\left(1+a e^{k x}\right)^{2}}+\frac{2 a k^{3} \sqrt{1+k^{2}}\left(1-a e^{k x}\right) e^{k x}}{\left(1+a e^{k x}\right)^{3}} t \\
& +\int_{0}^{t}(x-t)\left(\frac{\partial^{2} u_{n}}{\partial t^{2}}-\left(\tilde{u}_{n}\right)_{x x}-\left(\tilde{u}_{n}\right)_{x x x x}-3 \sum_{n=0}^{\infty} B_{n}\right) d t .
\end{aligned}
$$

The following approximants are obtained:

$$
\begin{aligned}
& u_{0}(x, t)=\frac{2 e^{x}}{\left(1+e^{x}\right)^{2}} \\
& u_{1}(x, t)=\frac{2 e^{x}}{\left(1+e^{x}\right)^{2}}+\frac{2 a k^{3} \sqrt{1+k^{2}}\left(1-a e^{k x}\right) e^{k x}}{\left(1+a e^{k x}\right)^{3}} t+\frac{2 e^{x}\left(1-4 e^{x}+e^{2 x}\right)}{\left(1+e^{x}\right)^{4}} t^{2}, \\
& u_{2}(x, t)=\frac{2 e^{x}}{\left(1+e^{x}\right)^{2}}+\frac{2 a k^{3} \sqrt{1+k^{2}}\left(1-a e^{k x}\right) e^{k x}}{\left(1+a e^{k x}\right)^{3}} t+\frac{2 e^{x}\left(1-4 e^{x}+e^{2 x}\right)}{\left(1+e^{x}\right)^{4}} t^{2} \\
& -\frac{2 \sqrt{2} e^{x}\left(-1+e^{x}\right)\left(1-10 e^{x}+e^{2 x}\right)}{3\left(1+e^{x}\right)^{5}} t^{3} \\
& +\frac{e^{x}\left(1-4 e^{x}+e^{2 x}\right)\left(1-44 e^{x}+78 e^{2 x}-44 e^{3 x}+e^{4 x}\right)}{3\left(1+e^{x}\right)^{8}} t^{4}, \\
& u_{3}(x, t)=\frac{2 e^{x}}{\left(1+e^{x}\right)^{2}}+\frac{2 a k^{3} \sqrt{1+k^{2}}\left(1-a e^{k x}\right) e^{k x}}{\left(1+a e^{k x}\right)^{3}} t+\frac{2 e^{x}\left(1-4 e^{x}+e^{2 x}\right)}{\left(1+e^{x}\right)^{4}} t^{2} \\
& -\frac{2 \sqrt{2} e^{x}\left(-1+e^{x}\right)\left(1-10 e^{x}+e^{2 x}\right)}{3\left(1+e^{x}\right)^{5}} t^{3} \\
& +\frac{e^{x}\left(1-4 e^{x}+e^{2 x}\right)\left(1-44 e^{x}+78 e^{2 x}-44 e^{3 x}+e^{4 x}\right)}{3\left(1+e^{x}\right)^{8}} t^{4} \\
& -\frac{\sqrt{2} e^{x}\left(-1+e^{x}\right)\left(1-56 e^{x}+246 e^{2 x}-56 e^{3 x}+e^{4 x}\right)}{15\left(1+e^{x}\right)^{7}} t^{5} \\
& +\frac{e^{x}\left(1-452 e^{x}+19149 e^{2 x}-207936 e^{3 x}+807378 e^{4 x}-1256568 e^{5 x}\right)}{45\left(1+e^{x}\right)^{12}} t^{6} \\
& +\frac{e^{x}\left(807378 e^{6 x}-207936 e^{7 x}+19149 e^{8 x}-452 e^{9 x}+e^{10 x}\right)}{45\left(1+e^{x}\right)^{12}} t^{6},
\end{aligned}
$$


Table 5: (Error estimates.) The absolute error between the exact and the series solutions. Higher accuracy can be obtained by introducing some more components of the series solution.

\begin{tabular}{lcccccc}
\hline$x_{i}$ & \multicolumn{5}{c}{$t_{j}$} & \\
& 0.01 & 0.02 & 0.04 & 0.1 & 0.2 & 0.5 \\
\hline-1 & $2.80886 E-14$ & $1.79667 E-12$ & $1.15235 E-10$ & $2.83355 E-8$ & $1.83899 E-6$ & $4.74681 E-4$ \\
-0.8 & $6.27276 E-14$ & $4.01362 E-12$ & $2.57471 E-10$ & $6.33178 E-8$ & $4.10454 E-6$ & $1.04489 E-3$ \\
-0.6 & $6.08402 E-14$ & $3.90188 E-12$ & $2.25663 E-10$ & $6.18024 E-8$ & $4.02299 E-6$ & $1.03093 E-3$ \\
-0.4 & $1.16573 E-14$ & $7.41129 E-13$ & $4.82756 E-11$ & $1.23843 E-8$ & $8.53800 E-6$ & $2.46302 E-4$ \\
-0.2 & $5.53446 E-14$ & $3.53395 E-12$ & $2.25663 E-10$ & $5.47485 E-8$ & $3.47264 E-6$ & $8.35783 E-4$ \\
0 & $8.63198 E-14$ & $5.53357 E-12$ & $2.54174 E-10$ & $8.65197 E-8$ & $5.54893 E-6$ & $1.37353 E-3$ \\
0.2 & $5.56222 E-14$ & $3.55044 E-12$ & $2.27779 E-10$ & $5.60362 E-8$ & $3.63600 E-6$ & $9.29612 E-4$ \\
0.4 & $1.14353 E-14$ & $7.14928 E-13$ & $4.49107 E-11$ & $1.03370 E-8$ & $5.93842 E-7$ & $9.61260 E-5$ \\
0.6 & $6.06182 E-14$ & $3.87551 E-12$ & $2.47218 E-10$ & $5.97562 E-8$ & $3.76275 E-6$ & $8.79002 E-4$ \\
0.8 & $6.23945 E-14$ & $3.99519 E-12$ & $2.55127 E-10$ & $6.18881 E-8$ & $3.92220 E-6$ & $9.36404 E-4$ \\
1 & $2.79776 E-14$ & $1.78946 E-12$ & $1.14307 E-10$ & $2.77684 E-8$ & $1.76607 E-6$ & $4.28986 E-4$ \\
\hline
\end{tabular}

The series solution is given as

$$
\begin{aligned}
u(x, t)= & \frac{2 e^{x}}{\left(1+e^{x}\right)^{2}}+\frac{2 a k^{3} \sqrt{1+k^{2}}\left(1-a e^{k x}\right) e^{k x}}{\left(1+a e^{k x}\right)^{3}} t+\frac{2 e^{x}\left(1-4 e^{x}+e^{2 x}\right)}{\left(1+e^{x}\right)^{4}} t^{2} \\
& -\frac{2 \sqrt{2} e^{x}\left(-1+e^{x}\right)\left(1-10 e^{x}+e^{2 x}\right)}{3\left(1+e^{x}\right)^{5}} t^{3} \\
& +\frac{e^{x}\left(1-4 e^{x}+e^{2 x}\right)\left(1-44 e^{x}+78 e^{2 x}-44 e^{3 x}+e^{4 x}\right)}{3\left(1+e^{x}\right)^{8}} t^{4} \\
& +\frac{8 e^{2 x}\left(1-10 e^{x}+20 e^{2 x}-10 e^{3 x}+e^{4 x}\right)}{\left(1+e^{x}\right)^{8}} t^{4} \\
& -\frac{\sqrt{2} e^{x}\left(-1+e^{x}\right)\left(1-56 e^{x}+246 e^{2 x}-56 e^{3 x}+e^{4 x}\right)}{15\left(1+e^{x}\right)^{7}} t^{5} \\
& +\frac{e^{x}\left(1-452 e^{x}+19149 e^{2 x}-207936 e^{3 x}+807378 e^{4 x}-1256568 e^{5 x}\right)}{45\left(1+e^{x}\right)^{12}} t^{6} \\
& +\frac{e^{x}\left(807378 e^{6 x}-207936 e^{7 x}+19149 e^{8 x}-452 e^{9 x}+e^{10 x}\right)}{45\left(1+e^{x}\right)^{12}} t^{6}+\cdots
\end{aligned}
$$

Example 6.4 (see $[32,51])$. Consider the following singularly perturbed sixth-order Boussinesq equation:

$$
u_{t t}=u_{x x}+\left(u^{2}\right)_{x x}-u_{x x x x}+\frac{1}{2} u_{x x x x x x}
$$

with initial conditions

$$
u(x, 0)=-\frac{105}{169} \operatorname{sech}^{4}\left(\frac{x}{\sqrt{26}}\right), \quad u_{t}(x, 0)=\frac{-210 \sqrt{194 / 13} \operatorname{sech}^{4}(x / \sqrt{26}) \tanh (x / \sqrt{26})}{2197} .
$$




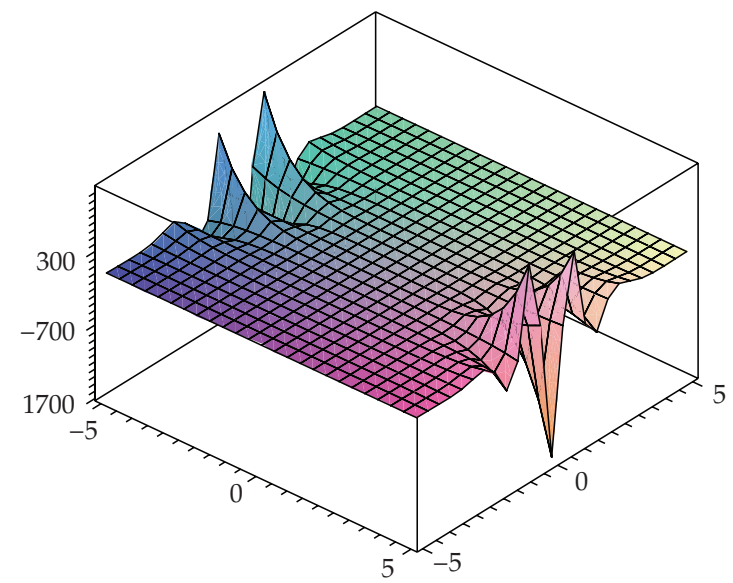

Figure 2

The exact solution of the problem is given as

$$
u(x, t)=-\frac{105}{169} \sec h^{4}\left[\sqrt{\frac{1}{26}}\left(x-\sqrt{\frac{97}{169}} t\right)\right]
$$

Applying the modified variational iteration method, we obtain

$$
\begin{aligned}
u_{n+1}(x, t)=- & \frac{105}{169} \sec h^{4}\left(\frac{x}{\sqrt{26}}\right)+\frac{-210 \sqrt{194 / 13} \sec ^{4}(x / \sqrt{26}) \tanh (x / \sqrt{26})}{2197} t \\
& +\int_{0}^{t} \lambda\left(\frac{\partial^{2} u_{n}}{\partial t^{2}}-\left(\tilde{u}_{n}\right)_{x x}+\left(\tilde{u}_{n}\right)_{x x x x}-\frac{1}{2}\left(\tilde{u}_{n}\right)_{x x x x x x}+\sum_{n=0}^{\infty} b_{n}\right) d t
\end{aligned}
$$

Making the correct functional stationary, the Lagrange multiplier can be identified as $\lambda=x-t$, consequently

$$
\begin{aligned}
u_{n+1}(x, t)= & -\frac{105}{169} \operatorname{sech}\left(\frac{x}{\sqrt{26}}\right)+\frac{-210 \sqrt{194 / 13} \sec h^{4}(x / \sqrt{26}) \tanh (x / \sqrt{26})}{2197} t \\
& +\int_{0}^{t}(x-t)\left(\frac{\partial^{2} u_{n}}{\partial t^{2}}-\left(\tilde{u}_{n}\right)_{x x}+\left(\tilde{u}_{n}\right)_{x x x x}-\frac{1}{2}\left(\tilde{u}_{n}\right)_{x x x x x x}+\sum_{n=0}^{\infty} B_{n}\right) d t
\end{aligned}
$$


where $B_{n}$ are Adomian's polynomials for nonlinear operator $F(u)=u^{2}(x)$ and can be generated for all types of nonlinearities according to the algorithm developed in [42-44, 51]. Consequently, the following approximants are obtained:

$$
\begin{aligned}
u_{0}(x, t)= & -\frac{105}{169} \operatorname{sech}^{4}\left(\frac{x}{\sqrt{26}}\right), \\
u_{1}(x, t)= & -\frac{105}{169} \operatorname{sech}^{4}\left(\frac{x}{\sqrt{26}}\right)-\frac{105 \sqrt{194 / 13} \sec h^{6}(x / \sqrt{26}) \sinh (\sqrt{2} x / \sqrt{13})}{2197} t \\
& -\frac{105}{371293}\left(-291+194 \cosh \left(\frac{\sqrt{2} x}{\sqrt{13}}\right)\right) \sec h^{6} \frac{x}{\sqrt{26}} t^{2}, \\
u_{2}(x, t)= & -\frac{105}{169} \sec h^{4}\left(\frac{x}{\sqrt{26}}\right)-\frac{105 \sqrt{194 / 13} \sec h^{6}(x / \sqrt{26}) \sinh (\sqrt{2} x / \sqrt{13})}{2197} t \\
& -\frac{105}{371293}\left(-291+194 \cosh \left(\frac{\sqrt{2} x}{\sqrt{13}}\right)\right) \sec h^{6} \frac{x}{\sqrt{26}} t^{2} \\
& +\frac{395 \sec h^{7}(x / \sqrt{26})}{52206766144}\left(10816 \sqrt{2522} \sinh \frac{x}{\sqrt{26}}-1664 \sqrt{2522} \sinh \frac{3 x}{\sqrt{26}}\right) t^{3} \\
& +\left(-334200 \sec h^{5}\left(\frac{x}{\sqrt{26}}\right)+354247 \cosh \left(\frac{2}{\sqrt{13}} x\right) \sec h^{5}\left(\frac{x}{\sqrt{26}}\right)\right. \\
& \left.-47164 \cosh \left(\frac{2 \sqrt{2}}{\sqrt{13}} x\right) \sec h^{5}\left(\frac{x}{\sqrt{26}}\right)\right) t^{4} \\
& +\left(3201 \cosh ^{3}\left(\frac{3 \sqrt{2}}{\sqrt{13}} x\right) \sec h^{5}\left(\frac{x}{\sqrt{26}}\right)-388 \cosh \left(\frac{4 \sqrt{2}}{\sqrt{13}} x\right) \sec h^{5}\left(\frac{x}{\sqrt{26}}\right)\right) t^{4}+\cdots,
\end{aligned}
$$

The series solution is obtained as

$$
\begin{aligned}
u(x, t)= & -\frac{105}{169} \operatorname{sech}^{4}\left(\frac{x}{\sqrt{26}}\right)-\frac{105 \sqrt{194 / 13} \operatorname{sech}^{6}(x / \sqrt{26}) \sinh (\sqrt{2} x / \sqrt{13})}{2197} t \\
& -\frac{105}{371293}\left(-291+194 \cosh \left(\frac{\sqrt{2} x}{\sqrt{13}}\right)\right) \operatorname{sech} h^{6} \frac{x}{\sqrt{26}} t^{2} \\
& +\frac{395 \sec h^{7}(x / \sqrt{26})}{52206766144}\left(10816 \sqrt{2522} \sinh \frac{x}{\sqrt{26}}-1664 \sqrt{2522} \sinh \frac{3 x}{\sqrt{26}}\right) t^{3} \\
& +\left(-334200 \operatorname{sech}\left(\frac{x}{\sqrt{26}}\right)+354247 \cosh \left(\frac{2}{\sqrt{13}} x\right) \sec h^{5}\left(\frac{x}{\sqrt{26}}\right)\right. \\
& \left.\quad-47164 \cosh \left(\frac{2 \sqrt{2}}{\sqrt{13}} x\right) \sec h^{5}\left(\frac{x}{\sqrt{26}}\right)\right) t^{4} \\
& +\left(3201 \cosh ^{3}\left(\frac{3 \sqrt{2}}{\sqrt{13}} x\right) \sec h^{5}\left(\frac{x}{\sqrt{26}}\right)-388 \cosh \left(\frac{4 \sqrt{2}}{\sqrt{13}} x\right) \sec h^{5}\left(\frac{x}{\sqrt{26}}\right)\right) t^{4}+\cdots
\end{aligned}
$$


Table 6: The absolute error between the exact and the series solutions. Higher accuracy can be obtained by introducing some more components of the series solution.

\begin{tabular}{lcccccc}
\hline$x_{i}$ & 0.01 & 0.02 & 0.04 & 0.1 & 0.2 & 0.5 \\
\hline-1 & $7.77156 E-16$ & $1.36557 E-14$ & $8.57869 E-13$ & $2.09264 E-10$ & $1.33823 E-8$ & $3.25944 E-6$ \\
-0.8 & $1.11022 E-16$ & $1.99840 E-15$ & $1.12688 E-13$ & $2.73880 E-11$ & $1.74288 E-9$ & $4.14094 E-7$ \\
-0.6 & $2.22045 E-16$ & $1.09912 E-14$ & $7.28861 E-13$ & $1.78030 E-10$ & $1.14025 E-8$ & $2.79028 E-6$ \\
-0.4 & $1.11022 E-16$ & $2.32037 E-14$ & $1.50302 E-12$ & $3.67002 E-10$ & $2.34944 E-8$ & $5.74091 E-6$ \\
-0.2 & $6.66134 E-16$ & $3.23075 E-14$ & $2.04747 E-12$ & $4.99918 E-10$ & $3.19983 E-9$ & $7.81509 E-6$ \\
0 & $4.44089 E-16$ & $3.49720 E-14$ & $2.24365 E-12$ & $5.47741 E-10$ & $3.50559 E-8$ & $8.55935 E-6$ \\
0.2 & $5.55112 E-16$ & $3.19744 E-14$ & $2.04714 E-12$ & $4.99820 E-10$ & $3.19858 E-8$ & $7.80749 E-6$ \\
0.4 & $3.33067 E-16$ & $2.32037 E-14$ & $1.50324 E-12$ & $3.66815 E-10$ & $2.34706 E-8$ & $5.72641 E-6$ \\
0.6 & $3.33067 E-16$ & $1.12133 E-14$ & $7.28528 E-12$ & $1.77772 E-10$ & $1.13695 E-8$ & $2.77022 E-6$ \\
0.8 & $3.33067 E-16$ & $1.99840 E-15$ & $1.13132 E-13$ & $2.76944 E-11$ & $1.78208 E-9$ & $4.41936 E-7$ \\
1 & $7.77156 E-16$ & $1.38778 E-14$ & $8.58313 E-13$ & $2.09593 E-10$ & $1.34244 E-8$ & $3.28504 E-6$ \\
\hline
\end{tabular}

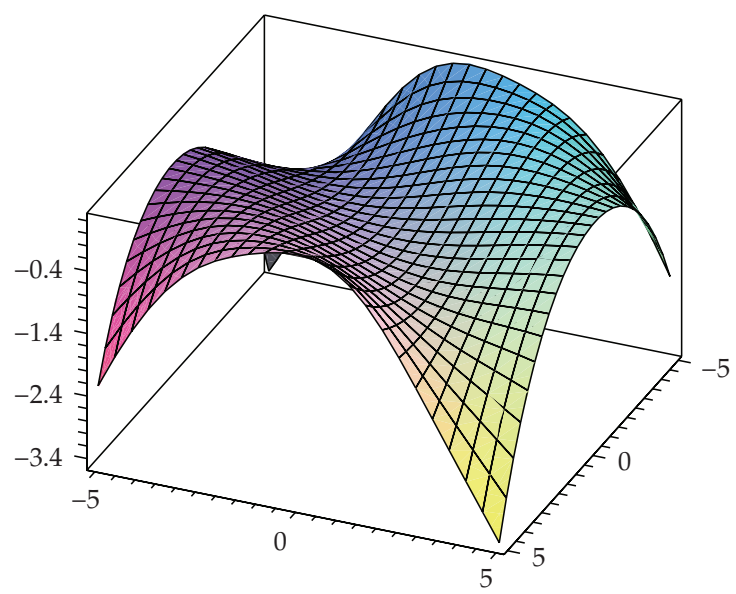

Figure 3

Example 6.5. Consider the following linear third-order dispersive $\mathrm{KdV}$ equation:

$$
u_{t}+2 u_{x}+u_{x x x}=0, \quad t>0,
$$

with initial condition

$$
u(x, 0)=\sin x
$$

The correct functional is given as

$$
u_{n+1}(x, t)=u_{n}(x, t)+\int_{0}^{x} \lambda(s)\left(\frac{\partial u_{n}}{\partial t}+2 \frac{\partial \tilde{u}_{n}}{\partial x}+\frac{\partial^{3} \tilde{u}_{n}}{\partial x^{3}}\right) d s
$$


Making the correct functional stationary, the Lagrange multiplier can be identified as $\lambda=-1$, consequently,

$$
u_{n+1}(x, t)=u_{n}(x, t)-\int_{0}^{x}\left(\frac{\partial u_{n}}{\partial t}+2 \frac{\partial \tilde{u}_{n}}{\partial x}+\frac{\partial^{3} \tilde{u}_{n}}{\partial x^{3}}\right) d s .
$$

Applying the modified variational iteration method,

$$
u_{n+1}(x, t)=u_{n}(x, t)-\int_{0}^{x}\left(\frac{\partial u_{n}}{\partial t}+2 \sum_{n=0}^{\infty} \frac{\partial \tilde{u}_{n}}{\partial x}+\sum_{n=0}^{\infty} \frac{\partial^{3} \tilde{u}_{n}}{\partial x^{3}}\right) d s
$$

Consequently, the following approximants are obtained:

$$
\begin{aligned}
& u_{0}(x, t)=\sin x \\
& u_{1}(x, t)=\sin x-t \cos x \\
& u_{2}(x, t)=\sin x\left(1-\frac{t^{2}}{2 !}\right)-t \cos x \\
& u_{3}(x, t)=\sin x\left(1-\frac{t^{2}}{2 !}\right)-\cos x\left(t-\frac{t^{3}}{3 !}\right),
\end{aligned}
$$

The series solution is given by

$$
u(x, t)=\sin x\left(1-\frac{t^{2}}{2 !}+\frac{t^{4}}{4 !}-\cdots\right)-\cos x\left(t-\frac{t^{3}}{3 !}+\frac{t^{5}}{5 !}+\cdots\right)
$$

and in a closed form by

$$
u(x, t)=\sin (x-t) .
$$

If we change the initial condition as $u(x, 0)=\cos x$, than the following closed-form solution will be obtained:

$$
u(x, t)=\cos (x-t)
$$

Example 6.6. Consider the following linear third-order dispersive $\mathrm{KdV}$ equation in a twodimensional space:

$$
u_{t}+u_{x x x}+u_{y y y}=0, \quad t>0
$$

with initial condition

$$
u(x, y, 0)=\cos (x+y)
$$

The correct functional is given as

$$
u_{n+1}(x, y, t)=u_{n}(x, y, t)+\int_{0}^{x} \lambda(s)\left(\frac{\partial u_{n}}{\partial t}+2 \frac{\partial^{3} \tilde{u}_{n}}{\partial x^{3}}+\frac{\partial^{3} \tilde{u}_{n}}{\partial y^{3}}\right) d s
$$




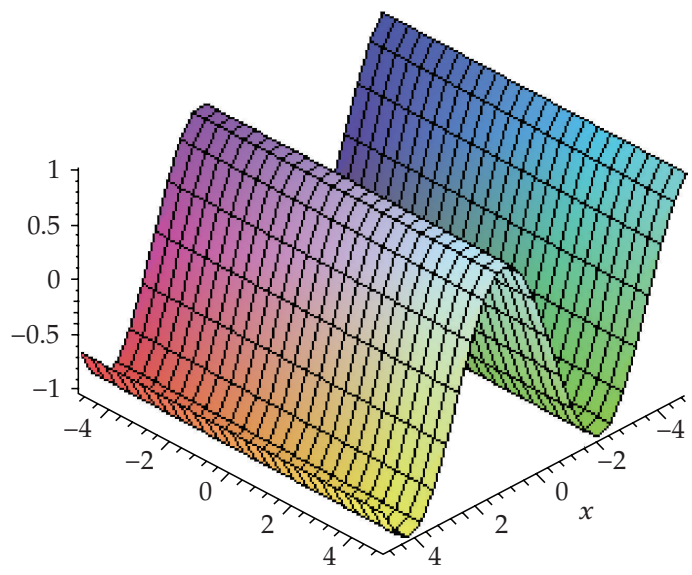

Figure 4

Making the correct functional stationary, the Lagrange multiplier can be identified as $\lambda=-1$, consequently,

$$
u_{n+1}(x, y, t)=u_{n}(x, y, t)-\int_{0}^{x}\left(\frac{\partial u_{n}}{\partial t}+2 \frac{\partial^{3} \tilde{u}_{n}}{\partial x^{3}}+\frac{\partial^{3} \tilde{u}_{n}}{\partial y^{3}}\right) d s .
$$

Applying the modified variational iteration method,

$$
u_{n+1}(x, y, t)=u_{n}(x, y, t)-\int_{0}^{x}\left(\frac{\partial u_{n}}{\partial t}+2 \sum_{n=0}^{\infty} \frac{\partial^{3} \tilde{u}_{n}}{\partial x^{3}}+\sum_{n=0}^{\infty} \frac{\partial^{3} \tilde{u}_{n}}{\partial y^{3}}\right) d s .
$$

Consequently, the following approximants are obtained:

$$
\begin{aligned}
& u_{0}(x, y, t)=\cos (x+y), \\
& u_{1}(x, y, t)=\cos (x+y)-2 t \sin (x+y), \\
& u_{2}(x, y, t)=\cos (x+y)\left(1-\frac{(2 t)^{2}}{2 !}\right)-(2 t) \sin (x+y), \\
& u_{3}(x, y, t)=\cos (x+y)\left(1-\frac{(2 t)^{2}}{2 !}\right)-\sin (x+y)\left((2 t)-\frac{(2 t)^{3}}{3 !}\right),
\end{aligned}
$$

The series solution is given by

$$
u(x, y, t)=\cos (x+y)\left(1-\frac{(2 t)^{2}}{2 !}+\cdots\right)-\sin (x+y)\left((2 t)-\frac{(2 t)^{3}}{3 !}+\cdots\right)
$$

and in a closed form by

$$
u(x, y, t)=\cos (x+y+2 t) .
$$

If we change the initial condition as $u(x, y, 0)=\sin (x+y)$, than the following closed-form solution will be obtained:

$$
u(x, y, t)=\sin (x+y+2 t)
$$




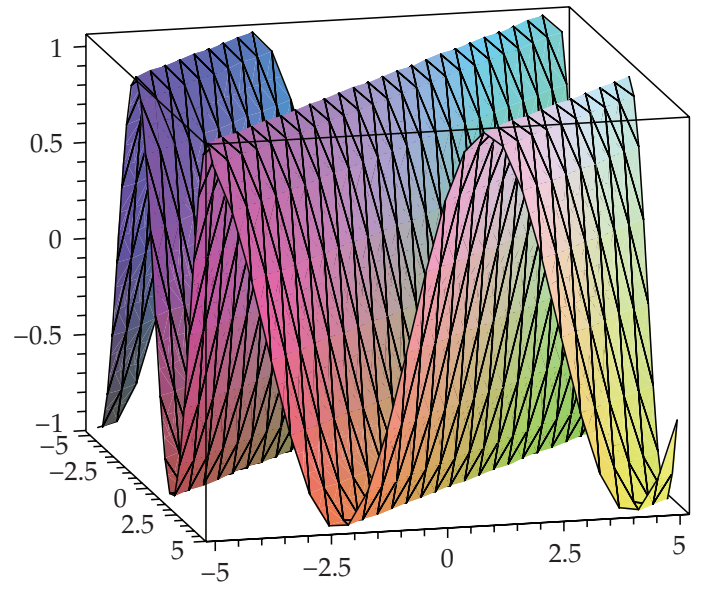

Figure 5: $(t=1)$.

Example 6.7 (see [42]). Consider the following singular fourth-order parabolic partial differential equation in two space variables:

$$
\frac{\partial^{2} u}{\partial t^{2}}+2\left(\frac{1}{x^{2}}+\frac{x^{4}}{6 !}\right) \frac{\partial^{4} u}{\partial x^{4}}+2\left(\frac{1}{y^{2}}+\frac{y^{4}}{6 !}\right) \frac{\partial^{4} u}{\partial y^{4}}=0
$$

with initial conditions

$$
u(x, y, 0)=0, \quad \frac{\partial u}{\partial t}(x, y, 0)=2+\frac{x^{6}}{6 !}+\frac{y^{6}}{6 !}
$$

and the boundary conditions

$$
\begin{array}{rlrl}
u\left(\frac{1}{2}, y, t\right)=\left(2+\frac{(0.5)^{6}}{6 !}+\frac{y^{6}}{6 !}\right) \sin t, & & u(1, y, t)=\left(2+\frac{1}{6 !}+\frac{y^{6}}{6 !}\right) \sin t \\
& \frac{\partial^{2} u}{\partial x^{2}}\left(\frac{1}{2}, y, t\right)=\frac{(0.5)^{4}}{24} \sin t, & \frac{\partial^{2} u}{\partial x^{2}}(1, y, t)=\frac{1}{24} \sin t \\
\frac{\partial^{2} u}{\partial y^{2}}\left(x, \frac{1}{2}, t\right)=\frac{(0.5)^{4}}{24} \sin t, & \frac{\partial^{2} u}{\partial y^{2}}(x, 1, t)=\frac{1}{24} \sin t .
\end{array}
$$

The correct functional is given as

$$
u_{n+1}(x, t)=u_{0}(x, t)+\int_{0}^{t} \lambda(\xi)\left(\frac{\partial^{2} u_{n}}{\partial t^{2}}+2\left(\frac{1}{x^{2}}+\frac{x^{4}}{6 !}\right) \frac{\partial^{4} \tilde{u}_{n}}{\partial x^{4}}+\left(2\left(\frac{1}{y^{2}}+\frac{y^{4}}{6 !}\right) \frac{\partial^{4} \tilde{u}_{n}}{\partial y^{4}}\right)\right) d \xi
$$

where $\tilde{u}_{n}$ is considered as a restricted variation. Making the above functional stationary, the Lagrange multiplier can be identified as $\lambda=\xi-t$, consequently,

$$
u_{n+1}(x, t)=u_{0}(x, t)+\int_{0}^{t}(\xi-t)\left(\frac{\partial^{2} u_{n}}{\partial t^{2}}+2\left(\frac{1}{x^{2}}+\frac{x^{4}}{6 !}\right) \frac{\partial^{4} \tilde{u}_{n}}{\partial x^{4}}+\left(2\left(\frac{1}{y^{2}}+\frac{y^{4}}{6 !}\right) \frac{\partial^{4} \tilde{u}_{n}}{\partial y^{4}}\right)\right) d \xi .
$$




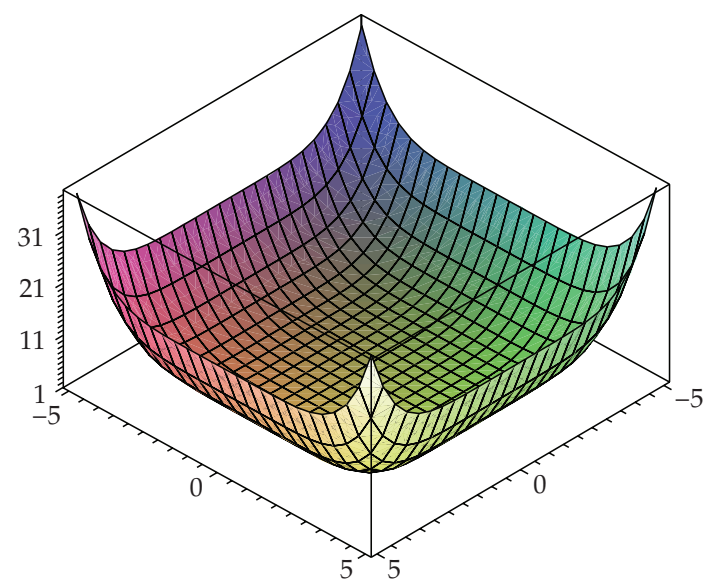

Figure 6: $(t=1)$.

Applying the modified variational iteration method, we have

$u_{n+1}(x, t)=u_{0}(x, t)+\int_{0}^{t}(\xi-t)\left(\frac{\partial^{2} u_{n}}{\partial t^{2}}+2\left(\frac{1}{x^{2}}+\frac{x^{4}}{6 !}\right) \sum_{n=0}^{\infty}\left(\frac{\partial^{4} \tilde{u}_{n}}{\partial x^{4}}\right)+\left(2\left(\frac{1}{y^{2}}+\frac{y^{4}}{6 !}\right) \sum_{n=0}^{\infty}\left(\frac{\partial^{4} \widetilde{u}_{n}}{\partial y^{4}}\right)\right)\right) d \xi$.

Consequently, the following approximants are obtained as:

$$
\begin{aligned}
& u_{0}(x, t)=\left(2+\frac{x^{6}}{6 !}+\frac{y^{6}}{6 !}\right) t \\
& u_{1}(x, t)=\left(2+\frac{x^{6}}{6 !}+\frac{y^{6}}{6 !}\right)\left(t-\frac{t^{3}}{3 !}\right) \\
& u_{2}(x, t)=\left(2+\frac{x^{6}}{6 !}+\frac{y^{6}}{6 !}\right)\left(t-\frac{t^{3}}{3 !}+\frac{t^{5}}{5 !}\right), \\
& u_{3}(x, t)=\left(2+\frac{x^{6}}{6 !}+\frac{y^{6}}{6 !}\right)\left(t-\frac{t^{3}}{3 !}+\frac{t^{5}}{5 !}-\frac{t^{7}}{7 !}\right), \\
& u_{4}(x, t)=\left(2+\frac{x^{6}}{6 !}+\frac{y^{6}}{6 !}\right)\left(t-\frac{t^{3}}{3 !}+\frac{t^{5}}{5 !}-\frac{t^{7}}{7 !}+\frac{t^{9}}{9 !}\right),
\end{aligned}
$$

The solution is given by

$$
u(x, y, t)=\left(2+\frac{x^{6}}{6 !}+\frac{y^{6}}{6 !}\right)\left(t-\frac{t^{3}}{3 !}+\frac{t^{5}}{5 !}-\frac{t^{7}}{7 !}+\frac{t^{9}}{9 !}+\cdots\right)=\left(2+\frac{x^{6}}{6 !}+\frac{y^{6}}{6 !}\right) \sin t
$$

Remark 6.8. It is worth mentioning that Ghorbani and Saberi-Nadjafi [49] and Ghorbani [50] introduced He polynomials which are compatible to Adomian's polynomials, are easier to calculate, and hence make the solution procedure simpler. 


\section{Conclusion}

In this paper, we applied the modified variational iteration method (MVIM) for solving singular and nonsingular initial and boundary value problems. The proposed technique is applied on boundary layer problem, unsteady flow of gas, Boussinesq equations, third-order dispersive and fourth-order parabolic partial differential equations. The Pade approximants were employed in order to make the work more concise and for better understanding of the solution behavior. It may be concluded that the proposed frame work is very powerful and efficient in finding the analytical solutions for singular and nonsingular boundary value problems. The method gives more realistic series solutions that converge very rapidly in physical problems.

\section{Acknowledgments}

The authors are highly grateful to the referee for his/her very constructive comments. The authors also would like to thank Dr. S. M. Junaid Zaidi, Rector CIIT for providing the excellent research facilities and environment. The authors are grateful to Asif Waheed for drawing the figures and useful comments. This research is supported by HEC research Grant.

\section{References}

[1] S. Abbasbandy, "A new application of He's variational iteration method for quadratic Riccati differential equation by using Adomian's polynomials," Journal of Computational and Applied Mathematics, vol. 207, no. 1, pp. 59-63, 2007.

[2] S. Abbasbandy, "Numerical solution of non-linear Klein-Gordon equations by variational iteration method," International Journal for Numerical Methods in Engineering, vol. 70, no. 7, pp. 876-881, 2007.

[3] S. Abbasbandy, "Numerical method for non-linear wave and diffusion equations by the variational iteration method," International Journal for Numerical Methods in Engineering, vol. 73, no. 12, pp. 18361843, 2008.

[4] M. A. Abdou and A. A. Soliman, "New applications of variational iteration method," Physica D, vol. 211, no. 1-2, pp. 1-8, 2005.

[5] J. Biazar and H. Ghazvini, "He's variational iteration method for fourth-order parabolic equations," Computers \& Mathematics with Applications, vol. 54, no. 7-8, pp. 1047-1054, 2007.

[6] J.-H. He, "Homotopy perturbation technique," Computer Methods in Applied Mechanics and Engineering, vol. 178, no. 3-4, pp. 257-262, 1999.

[7] J.-H. He, "Homotopy perturbation method for solving boundary value problems," Physics Letters A, vol. 350, no. 1-2, pp. 87-88, 2006.

[8] J.-H. He, "Comparison of homotopy perturbation method and homotopy analysis method," Applied Mathematics and Computation, vol. 156, no. 2, pp. 527-539, 2004.

[9] J.-H. He, "Homotopy perturbation method for bifurcation of nonlinear problems," International Journal of Nonlinear Sciences and Numerical Simulation, vol. 6, no. 2, pp. 207-208, 2005.

[10] J.-H. He, "The homotopy perturbation method for nonlinear oscillators with discontinuities," Applied Mathematics and Computation, vol. 151, no. 1, pp. 287-292, 2004.

[11] J.-H. He, "A coupling method of a homotopy technique and a perturbation technique for non-linear problems," International Journal of Non-Linear Mechanics, vol. 35, no. 1, pp. 37-43, 2000.

[12] J.-H. He, "Some asymptotic methods for strongly nonlinear equations," International Journal of Modern Physics B, vol. 20, no. 10, pp. 1141-1199, 2006.

[13] J.-H. He and X.-H. Wu, "Construction of solitary solution and compacton-like solution by variational iteration method," Chaos, Solitons E Fractals, vol. 29, no. 1, pp. 108-113, 2006.

[14] J.-H. He, "Variational iteration method-some recent results and new interpretations," Journal of Computational and Applied Mathematics, vol. 207, no. 1, pp. 3-17, 2007. 
[15] J.-H. He and X.-H. Wu, "Variational iteration method: new development and applications," Computers $\mathcal{E}$ Mathematics with Applications, vol. 54, no. 7-8, pp. 881-894, 2007.

[16] J.-H. He, "The variational iteration method for eighth-order initial-boundary value problems," Physica Scripta, vol. 76, no. 6, pp. 680-682, 2007.

[17] J.-H. He, "Variational iteration method-a kind of non-linear analytical technique: some examples," International Journal of Non-Linear Mechanics, vol. 34, no. 4, pp. 699-708, 1999.

[18] J.-H. He, "Variational iteration method for autonomous ordinary differential systems," Applied Mathematics and Computation, vol. 114, no. 2-3, pp. 115-123, 2000.

[19] M. Inokuti, H. Sekine, and T. Mura, "General use of the Lagrange multiplier in nonlinear mathematical physics," in Variational Method in the Mechanics of Solids, S. Nemat-Naseer, Ed., pp. 156-162, Pergamon Press, New York, NY, USA, 1978.

[20] R. E. Kidder, "Unsteady flow of gas through a semi-infinite porous medium," Journal of Applied Mechanics, vol. 24, pp. 329-332, 1957.

[21] S. Momani and S. Abuasad, "Application of He's variational iteration method to Helmholtz equation," Chaos, Solitons \& Fractals, vol. 27, no. 5, pp. 1119-1123, 2006.

[22] S. Momani and V. S. Ertürk, "Solutions of non-linear oscillators by the modified differential transform method," Computers \& Mathematics with Applications, vol. 55, no. 4, pp. 833-842, 2008.

[23] S. T. Mohyud-Din, "A reliable algorithm for Blasius equation," in Proceedings of the International Conference of Mathematical Sciences (ICMS '07), pp. 616-626, Selangor, Malaysia, November 2007.

[24] M. A. Noor and S. T. Mohyud-Din, "Homotopy perturbation method for nonlinear higher-order boundary value problems," International Journal of Nonlinear Sciences and Numerical Simulation, vol. 9, no. 4, pp. 395-408, 2008.

[25] S. T. Mohyud-Din and M. A. Noor, "Homotopy perturbation method for solving fourth-order boundary value problems," Mathematical Problems in Engineering, vol. 2007, Article ID 98602, 15 pages, 2007.

[26] M. A. Noor and S. T. Mohyud-Din, "An efficient algorithm for solving fifth-order boundary value problems," Mathematical and Computer Modelling, vol. 45, no. 7-8, pp. 954-964, 2007.

[27] M. A. Noor and S. T. Mohyud-Din, "Homotopy perturbation method for solving sixth-order boundary value problems," Computers \& Mathematics with Applications, vol. 55, no. 12, pp. 2953-2972, 2008.

[28] M. A. Noor and S. T. Mohyud-Din, "Homotopy method for solving eighth order boundary value problems," Journal of Mathematical Analysis and Approximation Theory, vol. 1, no. 2, pp. 161-169, 2006.

[29] M. A. Noor and S. T. Mohyud-Din, "Approximate solutions of Flieral-Petviashivili equation and its variants," International Journal of Mathematics and Computer Science, vol. 2, no. 4, pp. 345-360, 2007.

[30] M. A. Noor and S. T. Mohyud-Din, "Variational iteration technique for solving higher order boundary value problems," Applied Mathematics and Computation, vol. 189, no. 2, pp. 1929-1942, 2007.

[31] M. A. Noor and S. T. Mohyud-Din, "An efficient method for fourth-order boundary value problems," Computers \& Mathematics with Applications, vol. 54, no. 7-8, pp. 1101-1111, 2007.

[32] M. A. Noor and S. T. Mohyud-Din, "Variational iteration method for solving higher-order nonlinear boundary value problems using He's polynomials," International Journal of Nonlinear Sciences and Numerical Simulation, vol. 9, no. 2, 2008.

[33] M. A. Noor and S. T. Mohyud-Din, "Variational iteration method for fifth-order boundary value problems using He's polynomials," Mathematical Problems in Engineering, vol. 2008, Article ID 954794, 12 pages, 2008.

[34] M. A. Noor and S. T. Mohyud-Din, "Variational iteration method for unsteady flow of gas through a porous medium using He's polynomials and Pade approximants," Computer and Mathmetics with Applications. In press.

[35] M. A. Noor and S. T. Mohyud-Din, "Solution of twelfth-order boundary value problems by variational iteration technique," Journal of Applied Mathematics and Computing. In press.

[36] M. A. Noor and S. T. Mohyud-Din, "Variational iteration decomposition method for solving eighthorder boundary value problems," Differential Equations and Nonlinear Mechanics, vol. 2007, Article ID 19529, 16 pages, 2007.

[37] M. A. Noor and S. T. Mohyud-Din, "Modified variational iteration method for solving fourth-order boundary value problems," Journal of Applied Mathematics and Computing. In press.

[38] M. A. Noor and S. T. Mohyud-Din, "Variational decomposition method for singular initial value problems," International Journal of Pure and Applied Mathematics. In press. 
[39] M. A. Noor and S. T. Mohyud-Din, "Variational iteration method for solving initial value problems of Bratu type," Applications and Applied Mathematics, vol. 3, pp. 89-99, 2008.

[40] M. A. Noor and S. T. Mohyud-Din, "Variational iteration decomposition method for solving sixthorder boundary value problems," International Journal of Nonlinear Sciences and Numerical Simulation. In press.

[41] J. I. Ramos, "On the variational iteration method and other iterative techniques for nonlinear differential equations," Applied Mathematics and Computation, vol. 199, no. 1, pp. 39-69, 2008.

[42] A.-M. Wazwaz, "Analytic treatment for variable coefficient fourth-order parabolic partial differential equations," Applied Mathematics and Computation, vol. 123, no. 2, pp. 219-227, 2001.

[43] A.-M. Wazwaz, "A study on a boundary-layer equation arising in an incompressible fluid," Applied Mathematics and Computation, vol. 87, no. 2-3, pp. 199-204, 1997.

[44] A.-M. Wazwaz, "The modified decomposition method applied to unsteady flow of gas through a porous medium," Applied Mathematics and Computation, vol. 118, no. 2-3, pp. 123-132, 2001.

[45] L. Xu, "He's homotopy perturbation method for a boundary layer equation in unbounded domain," Computers E Mathematics with Applications, vol. 54, no. 7-8, pp. 1067-1070, 2007.

[46] L. Xu, "Variational iteration method for solving integral equations," Computers $\mathcal{E}$ Mathematics with Applications, vol. 54, no. 7-8, pp. 1071-1078, 2007.

[47] J. P. Boyd, "Padé approximant algorithm for solving nonlinear ordinary differential equation boundary value problems on an unbounded domain ," Computers in Physics, vol. 11, no. 3, pp. 299-303, 1997.

[48] D. D. Ganji, G. A. Afrouzi, and R. A. Talarposhti, "Application of He's variational iteration method for solving the reaction-diffusion equation with ecological parameters," Computers \& Mathematics with Applications, vol. 54, no. 7-8, pp. 1010-1017, 2007.

[49] A. Ghorbani and J. Saberi-Nadjafi, "He's homotopy perturbation method for calculating adomian polynomials," International Journal of Nonlinear Sciences and Numerical Simulation, vol. 8, no. 2, pp. 229 232, 2007.

[50] A. Ghorbani, "Beyond Adomian polynomials: He polynomials," Chaos, Solitons E Fractals. In press.

[51] M. A. Hajji and K. Al-Khaled, "Analytic studies and numerical simulations of the generalized Boussinesq equation," Applied Mathematics and Computation, vol. 191, no. 2, pp. 320-333, 2007. 


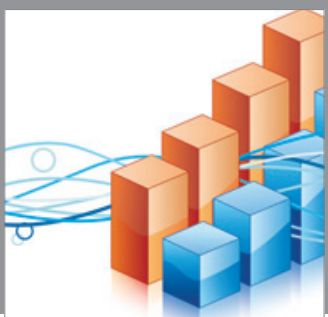

Advances in

Operations Research

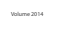

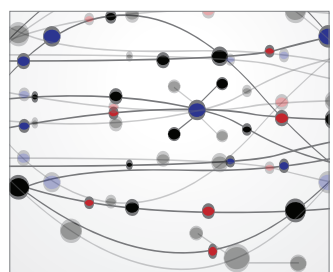

\section{The Scientific} World Journal
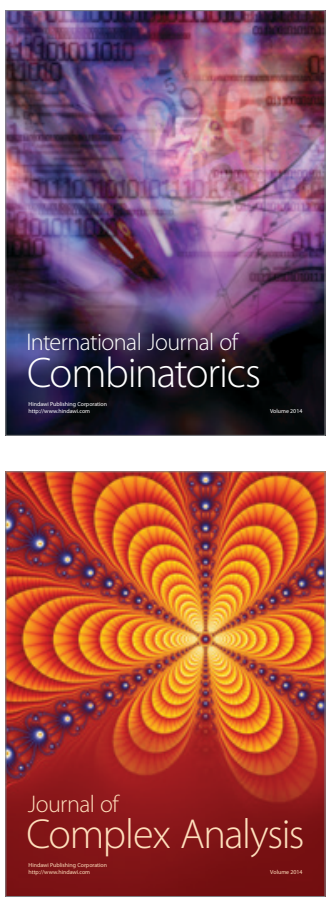

International Journal of

Mathematics and

Mathematical

Sciences
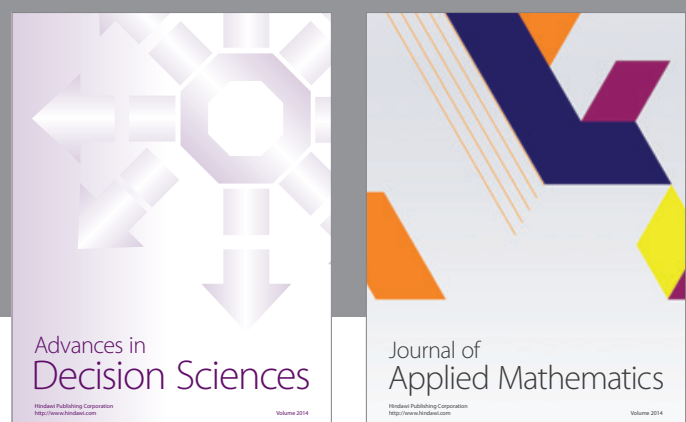

Journal of

Applied Mathematics
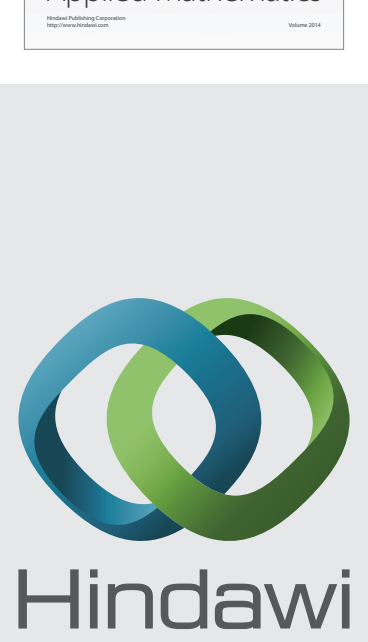

Submit your manuscripts at http://www.hindawi.com
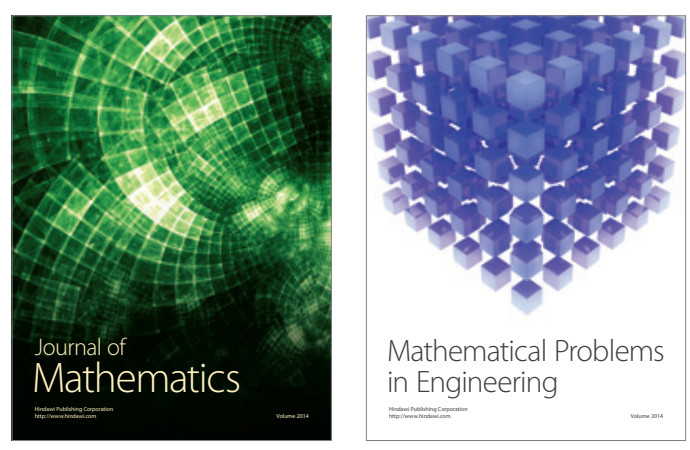

Mathematical Problems in Engineering
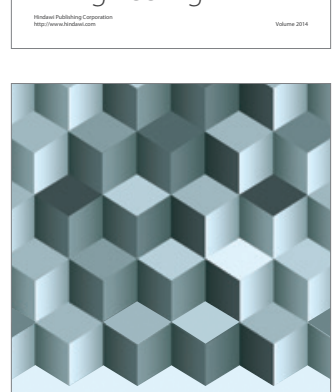

Journal of

Function Spaces
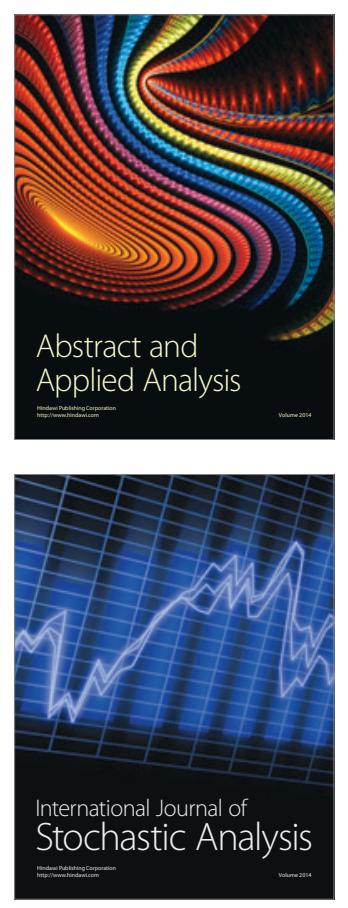

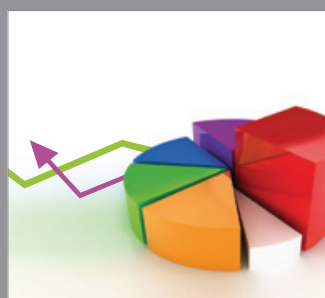

ournal of

Probability and Statistics

Promensencen
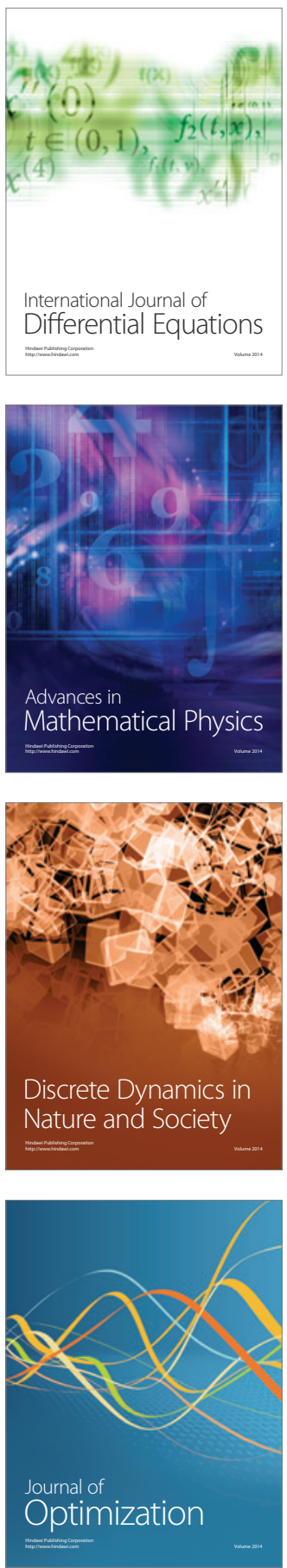